\title{
Comparação entre a lidocaína e a acupuntura no tratamento da taquicardia ventricular induzida com dopamina em equinos anestesiados com halotano
}

\author{
[Comparative study between lidocaine and acupunture in the treatment of ventricular tachycardia \\ induced by dopamine in horses anesthetized with halothane] \\ J.J. Cárdenas ${ }^{1}$, S.P.L. Luna $^{2 *}$, F. Teixeira Neto ${ }^{2}$, S.L. Beier ${ }^{3}$ \\ ${ }^{1}$ Médico veterinário autônomo \\ ${ }^{2}$ Faculdade de Medicina Veterinária e Zootecnia - UNESP \\ Distrito de Rubião Júnior s/n \\ 18618-000 - Botucatu - SP \\ ${ }^{3}$ Centro de Ciências Agroveterinárias - UDESC - Lages, SC
}

\begin{abstract}
RESUMO
Os efeitos da lidocaina e da acupuntura nos pontos bilaterais associados ao pericárdio 6 (Pc6-Neiguan) e ao coração 7 (C7-Shenmen), no tratamento da taquicardia ventricular (TV) induzida por dopamina em equinos anestesiados com halotano, foram avaliados e comparados. Seis equinos, distribuídos em três grupos: grupocontrole (GC), grupo tratado com acupuntura (GA) e grupo tratado com lidocaína (GL), foram anestesiados três vezes cada, com intervalo de uma semana entre cada anestesia. Avaliaram-se os parâmetros cardiovasculares (frequência cardíaca, pressão arterial e eletrocardiografia), os respiratórios (frequência respiratória, capnografía, saturação de hemoglobina e hemogasometria) e o escore de recuperação. A dose arritmogênica da dopamina (DAD) foi determinada a partir da infusão de $70 \mu \mathrm{g} / \mathrm{kg} / \mathrm{min}$ IV durante 10 minutos, sem interrupção, preenchendo o critério arritmogênico: quatro ou mais complexos ventriculares prematuros seguidos, com duração de pelo menos 15 segundos ou TV sustentada. O tempo médio de aparecimento da DAD ou da TV foi

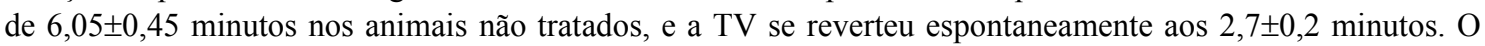
grupo tratado com acupuntura reverteu a TV no tempo médio de $1,8 \pm 0,2(\mathrm{P}<0,05)$ em relação ao grupo tratado com lidocaina $1,3 \pm 0,2(\mathrm{P}<0,01)$. Os tratamentos mostraram-se eficientes na reversão TV.
\end{abstract}

Palavras-chave: equino, taquicardia ventricular, halotano, acupuntura

\begin{abstract}
The effects of lidocaine and acupuncture in the associated bilateral points, i.e. pericardium 6 (Pc 6- Neiguan) and heart 7 (H7 - Shenmen), on the ventricular tachycardia (VT) induced by dopamine were evaluated in horses anesthetized with halothane. Six horses were distributed in three groups: control group (CG), acupuncture treated group $(A G)$, and lidocaine treated group $(L G)$. They were anesthetized three times each one using halothane with one week interval between each anesthesic procedure. Cardiovascular (heart rate, arterial pressure, and ECG) and respiratory (respiratory rate, capnometry, hemoglobin saturation, and blood gas analysis) parameters and recovery score were evaluated. The arrhythmogenic dose of dopamine (ADD) was determined by the infusion of $70 \mathrm{mg} / \mathrm{kg} / \mathrm{min}$ during 10 minutes without interruption fulfilling the arrhythmogenic criterion by the presence of four or more ectopic ventricular contractions during at least 15 seconds or maintained VT. The mean time for the occurrence of ADD or VT was $6.05 \pm 0.45$ minutes in no treated animals and the VT reverted spontaneously in $2.7 \pm 0.2$ minutes. The group treated with acupuncture reverted the VT in a mean time of $1.8 \pm 0.2(P<0.05)$ compared to the group treated with lidocaine $1.3 \pm 0.2$ $(P<0.01)$, both of them were efficacious for the treatment of VT.
\end{abstract}

Keywords: ventricular tachycardia, halothane, acupuncture

Recebido em 27 de junho de 2007

Aceito em 8 de abril de 2009

* Autor para correspondência (corresponding author)

E-mail: stelio@fmvz.unesp.br 


\section{INTRODUÇÃO}

Nos equinos, as complicações que ocorrem durante a anestesia geral, juntamente com a dificuldade na monitoração cardiorrespiratória, resultam em maior risco de óbito em relação a outras espécies animais.

Uma das manifestações comuns de problemas transanestésicos são as arritmias cardíacas. No cavalo, a taquicardia ventricular (TV) é reconhecida e definida no eletrocardiograma (ECG) pela presença de quatro ou mais complexos ventriculares prematuros (CVP) sucessivos (Patteson, 1996). O ritmo pode ser regular ou irregular, e a frequência cardíaca pode variar entre 120 e 200 nesta espécie (Muir e Hubbell, 1991). No equino, a TV pode ter um prognóstico desfavorável, mas, caso seja reconhecida rapidamente e as causas primárias estejam relacionadas com compensações independentes ao coração, a terapêutica antiarrítmica geralmente apresenta bons resultados (Patteson, 1996).

A indução de arritmias como a TV em cavalos sadios pode ser um bom modelo de estudo de uma arritmia em situações controladas. A simulação dessa situação permitiria reconhecer os diferentes mecanismos homeostásicos de compensação e/ou descompensação nesse tipo de alteração, além de possibilitar a avaliação de diferentes terapias, convencionais ou não.

Este estudo teve como objetivos estabelecer um modelo não invasivo, simples e replicável de indução de TV, determinar a dose arritmogênica da dopamina (DAD), avaliar os efeitos cardiorrespiratórios da TV induzida por dopamina em equinos anestesiados com halotano e comparar o efeito da lidocaina, como método convencional, em relação à acupuntura bilateral nos pontos associados: pericárdio 6 (Pc $6-$ Neiguan) e coração 7 (C 7 - Shenmen) no tratamento de TV nesta espécie.

\section{MATERIAL E MÉTODOS}

Foram utilizados seis equinos adultos, de diversas raças, com pesos entre 250 e $450 \mathrm{~kg}$ e idades entre quatro a nove anos, distribuídos aleatoriamente pelo método de quadrado latino e divididos em três grupos: grupo-controle (GC), grupo tratado com lidocaína (GL) e grupo tratado com acupuntura (GA). Após jejum alimentar sólido e líquido, os animais foram anestesiados e mantidos na respiração controlada com halotano.

Foram realizadas várias tentativas para padronização da dose arritmogênica da dopamina (DAD) em três estudos pilotos, no quais somente o terceiro foi considerado o modelo ideal para ser utilizado neste estudo. No primeiro, quatro equinos foram infundidos com dopamina nas doses de 30,40 e $50 \mu \mathrm{g} / \mathrm{kg} / \mathrm{min} \mathrm{IV}$, durante três minutos cada, a intervalos de 10 minutos. O tempo máximo entre infusões e intervalos foi de 30 minutos. No segundo, três equinos foram infundidos com dopamina na dose inicial de $60 \mu \mathrm{g} / \mathrm{kg} / \mathrm{min} \mathrm{IV}$, sem interrupção, em no máximo 10 minutos. Nos casos em que o critério arritmogênico não era preenchido, a dose da dopamina era incrementada de forma crescente em somatórios de $10 \mathrm{em} 10 \mu \mathrm{g} / \mathrm{kg} / \mathrm{min}$. Quando esse critério era preenchido, a infusão era interrompida. No terceiro, foi infundida uma dose inicial de $70 \mu \mathrm{g} / \mathrm{kg} / \mathrm{min}$ de dopamina IV, sem interrupção, em dois animais. Nesta dose, os animais preencheram o critério determinado como arritmogênico, e foi esta a escolhida para infundir como protocolo nos animais dos três grupos.

A determinação da DAD foi aplicada com as médias de todos os animais dos grupos estudados e calculada pela fórmula: DAD $(\mu \mathrm{g} / \mathrm{kg})=$ dose de infusão $(\mu \mathrm{g} / \mathrm{kg} / \mathrm{min}) \mathrm{x}$ tempo $(\mathrm{min})$ até o primeiro CVP que preencheu o critério. $\mathrm{O}$ protocolo experimental constou dos seguintes momentos: basal (M0): imediatamente após a estabilização da anestesia geral inalatória; M1: presença de TV sustentada ou preenchimento do critério arritmogênico; M2: tempo decorrido do final da administração de dopamina até a ausência da TV; M3: tempo decorrido do final da administração de dopamina até a normalização do ritmo e da frequência. A partir do M3, a anestesia foi mantida por mais 30 minutos.

O protocolo experimental constou dos seguintes tratamentos: GC, após a interrupção da infusão de dopamina (M1), os animais não receberam nenhum tratamento; GL, imediatamente após $\mathrm{M} 1$, os animais foram tratados com $2 \mathrm{mg} / \mathrm{kg}$ de cloridrato de lidocaina IV em forma de bolus; GA, imediatamente após M1, foram inseridas bilateralmente agulhas para acupuntura nos 
pontos: pericárdio 6 (neiguan), localizado na borda cranial da castanha, na face medial do membro torácico e coração 7 (shenmen), localizado na extremidade distal lateral do rádio, proximal ao carpo acessório, na inserção do tendão flexor carpo ulnar. As agulhas foram rotacionadas sempre por uma mesma pessoa até o desaparecimento da TV. A profundidade de inserção das agulhas foi de $1 \mathrm{~cm}$.

$\mathrm{Na}$ avaliação paramétrica, registraram-se parâmetros cardiovasculares (frequência cardíaca, eletrocardiografia e pressão arterial) e respiratórios (frequência respiratória, capnometria, oximetria de pulso e hemogasometria arterial); além do tempo até o aparecimento da $\mathrm{TV}$ ou preenchimento do critério arritmogênico, tempo de TV, tempo de início do aumento da onda T após o fím da TV, tempo de aumento da onda $\mathrm{T}$, bem como o tempo e a qualidade da recuperação segundo a escala de Taylor (1991). A análise estatística foi efetuada por meio do programa Graphpad Instat.

\section{RESULTADOS}

Dos três pilotos para determinar a DAD, o primeiro não preencheu o critério determinado como arritmogênico em nenhum dos animais avaliados. O segundo preencheu o critério em dois de três animais, sendo necessário o aumento da dose de 60 para $70 \mu \mathrm{g} / \mathrm{kg} / \mathrm{min}$ no animal correspondente à infusão insuficiente. A nova infusão atingiu o parâmetro esperado antes de 10 minutos. $\mathrm{O}$ terceiro preencheu o critério arritmogênico nos dois animais em 6,05 $\pm 0,45$ minutos e com uma DAD de $423 \pm 31,7 \mu \mathrm{g} / \mathrm{kg}$. A sequência de arritmias após a padronização da DAD foi similar para cada grupo (Tab. 1, Fig. 1).

Tabela 1. Sequência e tempo médio do aparecimento das diferentes alterações de ritmo e frequência cardíaca, observada a partir do início da infusão IV de dopamina na dose de $70 \mu \mathrm{g} / \mathrm{kg} / \mathrm{min}$, até o aparecimento da taquicardia ventricular (M1), em equinos anestesiados com halotano e não tratados (GC) ou tratados com lidocaína (GL) ou acupuntura (GA)

\begin{tabular}{|c|c|c|c|}
\hline Grupo & Animal & Tempo (min) & Arritmia \\
\hline \multirow{6}{*}{$\mathrm{GC}$} & 1 & \multirow{6}{*}{$1,55 \pm 0,24$} & BAV2, EV, TS, CVP, TV, T个, Inv T. \\
\hline & 2 & & $\mathrm{BAV} 2, \mathrm{TS}, \mathrm{TV}, \mathrm{TS}, \mathrm{T} \uparrow$ \\
\hline & 3 & & BAV2, CVP, TV, TS, T个 \\
\hline & 4 & & $\mathrm{EV}, \mathrm{TS}, \mathrm{TV}, \mathrm{TS}, \mathrm{T} \uparrow$ \\
\hline & 5 & & BAV2, TS, CVP, TV, TS, T个, Inv T. \\
\hline & 6 & & BAV2, CVP, TV, T个, Inv T. \\
\hline \multirow{6}{*}{ GL } & 1 & \multirow{6}{*}{$1,65 \pm 0,25$} & BAV2, EV, TS, TV, T个 \\
\hline & 2 & & $\mathrm{BS}, \mathrm{EV}, \mathrm{TS}, \mathrm{CVP}, \mathrm{TV}, \mathrm{T} \uparrow$ \\
\hline & 3 & & BS, BAV2, CVP, TV, T个 \\
\hline & 4 & & $\mathrm{BAV} 2, \mathrm{EV}, \mathrm{CVP}, \mathrm{TV}, \mathrm{T} \uparrow$ \\
\hline & 5 & & $\mathrm{TS}, \mathrm{CVP}, \mathrm{TV}, \mathrm{T} \uparrow$ \\
\hline & 6 & & BAV2, TS, CVP, TS, TV, T个 \\
\hline \multirow{6}{*}{ GA } & 1 & \multirow{6}{*}{$1,85 \pm 0,27$} & BAV2, EV, TS, CVP, TV, T个 \\
\hline & 2 & & $\mathrm{BAV} 2, \mathrm{TS}, \mathrm{CVP}, \mathrm{TV}, \mathrm{T} \uparrow$ \\
\hline & 3 & & $\mathrm{EV}, \mathrm{TS}, \mathrm{CVP}, \mathrm{TV}, \mathrm{T} \uparrow$ \\
\hline & 4 & & BAV2, TS, TV, T, $\uparrow$ Inv T. \\
\hline & 5 & & $\mathrm{BAV} 2, \mathrm{TS}, \mathrm{TV}, \mathrm{T} \uparrow$ \\
\hline & 6 & & $\mathrm{EV}, \mathrm{TS}, \mathrm{CVP}, \mathrm{TV}, \mathrm{T} \uparrow$ \\
\hline
\end{tabular}

BS: bradicardia sinusal; BAV2: bloqueio atrioventricular de $2^{\circ}$; TS: taquicardia sinusal; BS: bradicardia sinusal; CVP: complexo ventricular prematuro; EV: escape ventricular; TV: taquicardia ventricular; GC: grupo-controle; GL: grupo tratado com lidocaina; GA: grupo tratado com acupuntura; min: minutos, $\uparrow:$ aumento da onda referida; inv: inversão da onda referida; T: onda "T". 


\section{Cárdenas et al.}

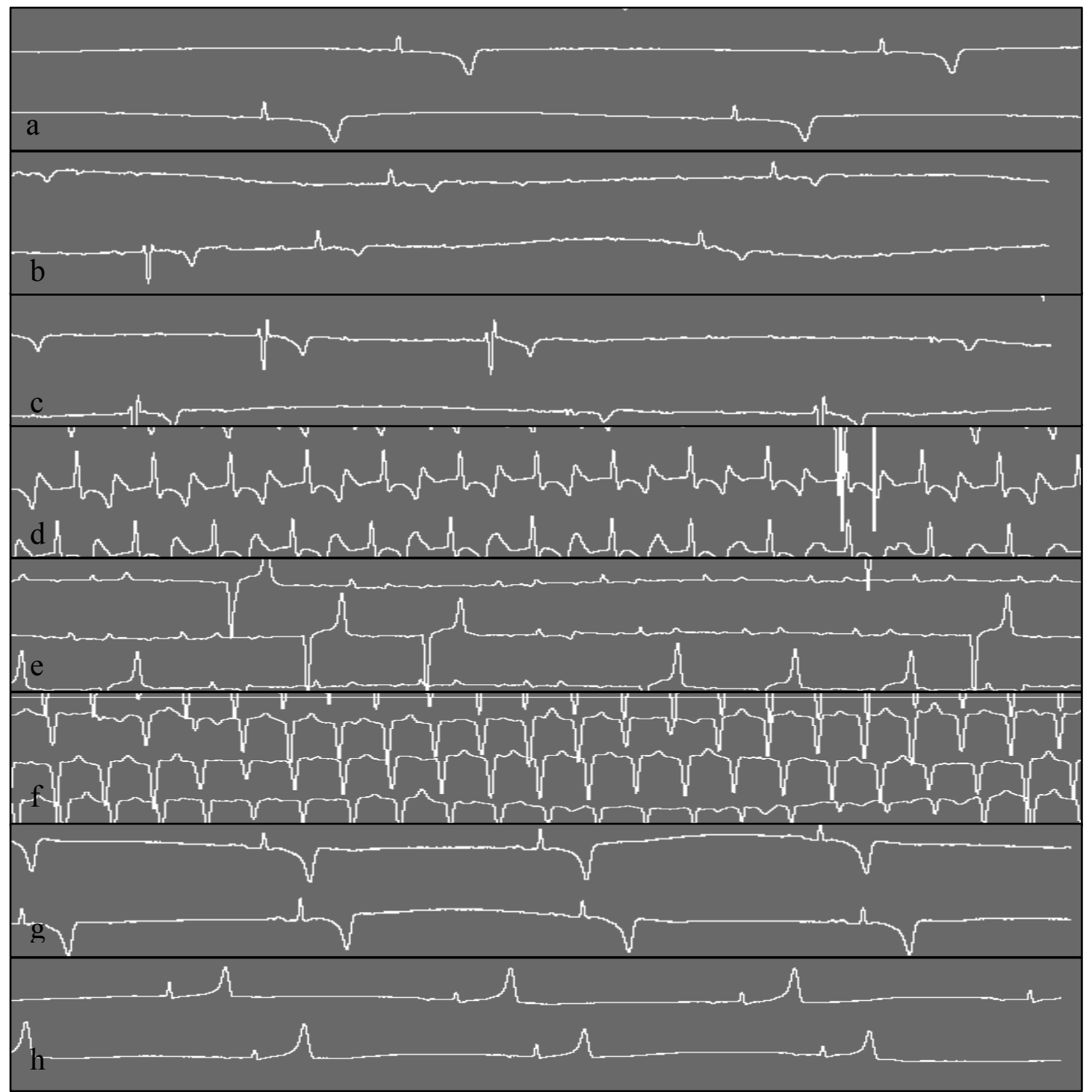

Figura 1. Sequência dos traçados eletrocardiográficos obtidos no módulo de aquisição de ECG-PC/TEB, na velocidade de $50 \mathrm{~mm} / \mathrm{seg}$, calibração $\mathrm{N}$, derivação aVL, em equinos de diferentes grupos. Verificam-se as ocorrências de: (a): bradicardia sinusal, (b): bloqueio atrioventricular de $2^{\circ}$ grau, (c): escape ventricular, (d): taquicardia sinusal, (e): complexos ventriculares prematuros, (f): taquicardia ventricular sustentada monomórfica, (g): aumento de onda " $\mathrm{T}$ "e (h): inversão da onda " $\mathrm{T}$ " com consequente aumento.

Para a frequência cardíaca, embora tenha havido aumento em cada momento de observação, não houve diferença significativa entre os grupos (Tab. 2). Da mesma forma, na pressão sistólica, na diastólica e na média, não foram observadas diferenças entre os grupos. O tempo transcorrido entre o início da infusão contínua de dopamina até o aparecimento da TV (M1) não foi diferente entre os grupos. No GC, o tempo de duração da taquicardia ventricular até o desaparecimento da arritmia (M1 a M2) de 2,7 $\pm 0,2$ minutos foi significativamente maior em relação ao GL e GA, 1,3 $\pm 0,2$ e 1,8 $\pm 0,2$ minutos, respectivamente. 
Tabela 2. Valores médios e desvios-padrão da frequência cardíaca em equinos anestesiados com halotano, submetidos à infusão contínua de dopamina intravenosa, não tratados (GC) ou tratados com lidocaína (GL) ou acupuntura (GA)

\begin{tabular}{cccc}
\hline Grupo & GC & GL & GA \\
\hline M0 & $28 \pm 5 \mathrm{c}$ & $30 \pm 7 \mathrm{~b}$ & $26 \pm 5 \mathrm{~b}$ \\
M1 & $144 \pm 32 \mathrm{a}$ & $151 \pm 36 \mathrm{a}$ & $142 \pm 53 \mathrm{a}$ \\
M2 & $82 \pm 23 \mathrm{~b}$ & $68 \pm 34 \mathrm{~b}$ & $79 \pm 60 \mathrm{ab}$ \\
M3 & $46 \pm 15 \mathrm{bc}$ & $68 \pm 34 \mathrm{~b}$ & $53 \pm 18 \mathrm{~b}$ \\
\hline
\end{tabular}

GC: grupo controle; GL: grupo tratado com lidocaina; GA: grupo tratado com acupuntura; M0: momento basal; M1: momento de aparecimento da taquicardia ventricular; M2: momento do fim da taquicardia ventricular; M3: normalização do ritmo e frequência.

Letras minúsculas distintas na coluna indicam diferença em relação aos momentos dentro de cada grupo.

Todos os animais dos três grupos apresentaram aumento na onda $\mathrm{T}$, acima de $25 \%$ da onda $\mathrm{R}$. Essa alteração ocorreu antes ou após a normalização do ritmo e da frequência cardíaca (M3), uma vez terminada a TV (M2). O tempo no aumento na onda $\mathrm{T}$, monitorado até 30 minutos após a anestesia, de 13,0 $\pm 11,15 \pm 6,5$ e $15 \pm 6,4$ minutos para GC, GL e GA, respectivamente, foi semelhante entre os grupos. O tempo médio de recuperação anestésica foi de $57 \pm 16$ para GC, $44 \pm 13,9$ para GL e $48 \pm 22,4$ para o GA. O escore de recuperação foi de 5 para nove animais $(50 \%), 4$ para seis $(33,3 \%)$ e 3 para três $(16,6 \%)$.

\section{DISCUSSÃO}

Alguns dos fatores importantes que dificultaram a determinação do método para padronizar a DAD foram os poucos estudos realizados com cavalos, bem como dos poucos disponíveis, com dopamina. Os modelos variados dos estudos também foram outra variável limitante. A seleção do cloridrato de dopamina foi devido à sua menor capacidade arritmogênica, em comparação ao isoprotenerol, à epinefrina e à dobutamina (Ueda et al., 1977), e pelo risco destas causar taquiarritmias irreversíveis com consequências danosas (Grandy et al., 1989; Gaynor et al., 1992; Gaynor et al., 1993).

Não foi possível determinar a DAD nas infusões seriadas de 30,40 e $50 \mu \mathrm{g} / \mathrm{kg} / \mathrm{min}$ de dopamina IV devido ao curto período de infusão associado ao longo período de repouso. Essa apreciação justifica-se quando comparada com os trabalhos de Sato et al. (1989), em que, mesmo na dose final de $15 \mu \mathrm{g} / \mathrm{kg} / \mathrm{min}$ de infusão de dopamina durante 30 minutos, não houve taquiarritmias em todos os animais. Segundo Robertson et al.
(1996), parece existir um importante efeito acumulativo de catecolaminas sistêmicas nos métodos de infusões que aumentam aritmeticamente, quando não se atinge o critério arritmogênico da catecolamina a ser estudada. Esse fator pode contribuir com o aumento no risco de apresentação de taquiarritmias irreversíveis, determinadas por estímulo permanente dos receptores adrenérgicos $\alpha 1$ e $\beta 1$, além do desequilíbrio autonômico que produz esta infusão.

Como segunda medida, uma troca de método para determinar a DAD melhorou a proximidade para atingir o critério considerado como arritmogênico, quando foi infundida a dose de $60 \mu \mathrm{g} / \mathrm{kg} / \mathrm{min}$ de dopamina IV em um tempo máximo de 10 minutos. Embora não tenha sido utilizado o critério considerado arritmogênico em $100 \%$ dos animais, o incremento de mais $10 \mu \mathrm{g} / \mathrm{kg} / \mathrm{min}$ satisfez essa condição. Dessa forma, a infusão de $70 \mu \mathrm{g} / \mathrm{kg} / \mathrm{min}$ foi efetiva para preencher o critério arritmogênico para todos os animais deste estudo.

A DAD de $423 \mu \mathrm{g} / \mathrm{kg}$, ao ser comparada com o estudo de Light e Hellyer (1993), cujo resultado foi de $105,6 \pm 16,3 \mu \mathrm{g} / \mathrm{kg}$, ao avaliarem o efeito da atropina na dose arritmogênica de dobutamina em cavalos utilizando o método de incremento aritmético, pode ser considerada alta. Isso pode ser justificado pela infusão, desde o início, de uma dose alta $(70 \mu \mathrm{g} / \mathrm{kg} / \mathrm{min})$ e pelo tempo prolongado que a infusão levou para preencher o critério arritmogênico desejado. Por outro lado, esse método minimizou o efeito acumulativo da catecolamina, pois com outros métodos de infusões repetidas, por meio de incremento aritmético das doses ou manutenção da infusão por mais tempo, a TV poderá ocorrer de forma 
mais rápida e diminuir a dose arritmogênica da catecolamina. Outro fator que influenciou $o$ resultado final da alta DAD foi o fato de a dopamina ser pouco arritmogênica e apresentar baixo inotropismo positivo, quando comparada a outras catecolaminas (Ueda et al., 1977).

Os tipos de arritmias supraventriculares relatadas por Robertson et al. (1996), com a infusão de $5 \mu \mathrm{g} / \mathrm{kg} / \mathrm{min}$ de dopamina em cavalos durante uma hora, não foram similares às arritmias supraventriculares deste estudo. Já as arritmias ventriculares na dose de $10 \mu \mathrm{g} / \mathrm{kg} / \mathrm{min}$ apresentaram maior semelhança, observando-se CVP e TV.

A dopamina em doses de infusão terapêutica (1$5 \mu \mathrm{g} / \mathrm{kg} / \mathrm{min}$ ) estimula receptores $\beta 1$ e $\beta 2$ com efeito inotrópico positivo, aumentando a frequência cardíaca, a resistência vascular periférica e a pressão arterial sanguínea. Doses intermediárias $(10 \mu \mathrm{g} / \mathrm{kg} / \mathrm{min})$ apresentam efeito mais pronunciado, enquanto doses maiores que $10 \mu \mathrm{g} / \mathrm{kg} / \mathrm{min}$ estimulam os receptores $\alpha 1$, produzindo vasoconstrição renal, diminuindo o fluxo sanguíneo dos rins e aumentando, ainda mais, a pressão arterial (Adam, 1980).

A tendência de estímulo dos baroreceptores em situação de hipertensão com bradicardia reflexa foi observada apenas em dois animais do GL. O bloqueio atrioventricular de segundo grau (BAV $2^{\circ}$ ) e o escape ventricular (EV) podem ser outra forma de estímulo vagal. Os fatores que poderiam ter contribuído em não se observar tão claramente a bradiarritmia supraventricular no começo da infusão, nestes animais, foi o espaçamento de tempo entre as colheitas dos dados (um minuto), que provavelmente foi longo para um volume de infusão de $70 \mu \mathrm{g} / \mathrm{kg} / \mathrm{min}$. Outro fator pode ser que, no ponto máximo de hipertensão, após a infusão, já teria sido infundida uma dose capaz de produzir arritmias ventriculares, ultrapassando o espaço de tempo para manifestação do tônus vagal e, consequentemente, a bradicardia sinusal.

A frequência cardíaca e a pressão arterial não foram diferentes entre os grupos; já entre os momentos, seria esperada elevação mais importante no M1, ao se atingir o critério arritmogênico da dopamina. Embora não tenha havido diferenças significativas entre os momentos M0 e M3 para os três grupos, nenhum valor de frequência cardíaca e alguns da pressão arterial voltaram aos seus valores basais. O tempo de aparecimento de taquicardia ventricular desde o começo da infusão com dopamina foi inferior aos 10 minutos, preenchendo o requisito exigido pelo modelo. $\mathrm{O}$ fato de os tempos serem parecidos entre os três grupos e de não haver nenhuma diferença significativa para este período entre os grupos aumentou a garantia de que o modelo possa ser replicável a outros estudos.

Embora o mecanismo de ação da lidocaína ainda não seja totalmente compreendido (Lynch et al., 1990), o bloqueio dos canais de sódio na membrana celular e a diminuição no automatismo das fibras de Purkinge são os fatores mais representativos no tratamento das arritmias ventriculares (Yashuda et al., 1991). Por esse motivo, os relatos de LeBlanc (1990) são compatíveis com a resposta que se obteve com o grupo tratado com lidocaína para tratar a arritmia ventricular, já que o tempo de desaparecimento da TV foi, aproximadamente, a metade do tempo transcorrido para esta ser revertida espontaneamente no grupo-controle.

A acupuntura nos pontos PC-6 e C-7 também foi efetiva para redução do tempo de desaparecimento da TV após a infusão de dopamina. Alguns autores sugerem que seu efeito no sistema cardiovascular ocorre por influência direta sobre o sistema autonômico, com inibição simpática, levando à correção de algumas situações patológicas, como as taquiarritmias cardíacas (Sternfeld et al., 1989). Outros correlacionam pontos específicos predeterminados com aumento da atividade vagal (Kazushi, 1997), para controlar a mesma situação.

Todos os animais começaram a apresentar aumento de onda $\mathrm{T}$ em um tempo aproximado de 10 a 11 minutos após o fim da TV. Embora o tempo de avaliação dos parâmetros cardiorrespiratórios tenha sido de 30 minutos após a finalização da TV, nem todos os animais apresentaram normalização da onda $T$ na finalização da anestesia. Muir e Hubbell (1991) expõem dois diagnósticos diferenciais que fazem com que a repolarização do miocárdio seja lenta: os níveis de potássio plasmáticos altos e a hipóxia do miocárdio. A indução experimental 
de hipercalemia em equinos produz alterações eletrocardiografias leves (6,2 milimoles/litro), com diminuição do tamanho da onda $\mathrm{P}$ e inversão da onda $T$. Já uma concentração plasmática maior (8-10 milimoles/litro) produz toxicidade no miocárdio, terminando com manifestação de arritmias, como contração atrial prematura, marcapasso migratório, $\mathrm{BAV} 2^{\circ}$, dissociação atrioventricular, complexos ventriculares prematuros (CVP), TV e parada cardíaca (Epstein, 1994). Neste estudo, a concentração plasmática de $\mathrm{K}^{+}$não se alterou, sendo que em M3 foi de 2,94 milimoles/litro. Dessa forma, as alterações ocorridas podem ser devido à hipóxia aguda de miocárdio.

$\mathrm{Na}$ maioria das espécies, a oxigenação miocárdica é mais eficiente na diástole ventricular. Nos cães, a TV reduz o tempo de preenchimento ventricular, aumenta o consumo de oxigênio (Teixeira Neto et al., 2001) e diminui o fluxo sanguíneo coronário em, aproximadamente, 60\% (Lunney e Ettinger, 1995). Essa situação não deve ser diferente no cavalo ao se atingir uma frequência cardíaca de 190 ou 193 batimentos/minuto em alguns dos animais avaliados no modelo experimental em M1.

Embora os tempos de duração da TV não tenham sido superiores a três minutos, inclusive para o grupo-controle, o tempo em que o traçado de ECG apresentou sinais compatíveis com hipóxia do miocárdio foi de 13 (GC) a 15 minutos (GL e GA) após o início do aumento da onda T. É interessante observar que o fato de o tempo de permanência do aumento da onda $\mathrm{T}$ para os grupos tratados $(15 \mathrm{~min}-\mathrm{GL}$ e GA) ter sido maior que o do grupo não tratado (13min - GC) descarta a possibilidade de relacionar o tempo de TV ou o aumento de frequência cardíaca com o tempo de hipóxia miocárdica que acontece nos pacientes submetidos a arritmia.

Embora o tempo de recuperação dos animais tratados tenha sido um pouco menor que o dos não tratados, estes valores mantiveram-se dentro do considerado normal para a anestesia inalatória com halotano em cavalos como de 60 - 90 minutos (Hubbell, 2004). Este resultado poderia sugerir que a alteração do débito cardíaco em função da TV não influenciou o tempo de recuperação dos animais infundidos com dopamina e/ou que a hipertensão sistêmica provavelmente manteve o fluxo sanguíneo e a perfusão muscular de forma homogênea para os animais dos grupos tratados e não tratados.

A partir dos resultados obtidos neste modelo experimental, conclui-se que: a DAD na velocidade de $70 \mu \mathrm{g} / \mathrm{kg} / \mathrm{min}$ foi de $423 \mu \mathrm{g} / \mathrm{kg}$; a dose de infusão da dopamina de $70 \mu \mathrm{g} / \mathrm{kg} / \mathrm{min}$ IV induz TV sustentada entre quatro e 10 minutos em cavalos anestesiados com halotano; tanto a lidocaína como a acupuntura bilateral nos pontos pericárdio 6 e coração 7, foram similarmente eficientes para o tratamento de TV em equinos anestesiados com halotano.

\section{REFERÊNCIAS BIBLIOGRÁFICAS}

ADAM. Ventricular arrhythmogenic dose of epinephrine in dogs and cats anesthetized with tiletamine/zolazepam and halothane Mod. Vet. Pract., v.54, p.37-42, 1980.

EPISTEIN, V. Relationship between potassium administration, hiperkalaemia and electrocardiogram: An experimental study. Equine Vet. J., v.32, p.453-456, 1994.

GAYNOR, J.S.; BEDNARSKI, R.M.; MUIR, W.W. Effects of xylazine on the arrhythmogenic dose of epinephrine in thiamylal/halothaneanesthetized horses. Am. J. Vet. Res., v.53, p.2350-2354, 1992.

GAYNOR, J.S.; BEDNARSKI, R.M.; MUIR, W.W. Effect of hypercapnia on the arrhythmogenic dose of epinephrine in horse anesthetized with guaifenesin, thyamylal sodium, and halothane. Am. J. Vet. Res., v.54, p.315-321, 1993.

GRANDY, J.L.; HODGSON, D.S.; DUNLOP, C.I. et al. Cardiopulmonary effects of ephedrine in halothane anesthetized horses. J. Vet. Pharmacol. Therap., v.12, p.389-396, 1989

HUBBELL, J.A. Anesthesia of the horse: Monitoring, Recovery, and Complications. AAEP Proc., v.50, p.291-295, 2004.

KAZUSHI, N. Decreased heart rate by acupuncture stimulation in humans via facilitation of cardiac vagal activity and suppression of cariac simphathetic nerve. Neurosci. Letters, v.227, p.166-168, 1997.

LeBLANC, P.H. Regional anesthesia. In: RIEBOLD, T.W. Principles and techniques of 
equine anesthesia. Vet. Clin. N. Am.: Equine Pract., v.6, p.693-704, 1990.

LIGHT, G.S.; HELLYER, P.W. Effects of atropine on arrythmogenic dose of dobutamine in xylazine-thiamylal-halothane-anesthetized horses. Am. J. Vet. Res., v.54, p.2099-2103, 1993.

LUNNEY, L.; ETTINGER, S.J. Cardiac arrhythymias. In: ETTINGER, S.J. Textbook of veterinary internal medicine. 4ed. Philadelphia: W.B. Saunders, 1995. p.959-995.

LYNCH, J.J.; HEANEY, L.A.; WALLACE, A.A. et. al. Failure of lidocaine to suppress lethal ischemic ventricular arrhythmias in a canine model of previous myocardial infarctation. $J$. Cardiov. Pharmacol., v.16, p.41-49, 1990.

MUIR, W.W.; HUBBELL, J. A. Equine anesthesia. monitoring and emergency therapy. St. Louis: Mosby Year Book, 1991. p.49-57.

PATTESON, M.W. Equine cardiology. Oxford: Blackwell Science, 1996. p.14, 115.

ROBERTSON, S.A. Metabolic, dopamine, hormonal, and hemodinamic changes during dopamine infusions in halothane anesthetizied horses. Vet. Anesth., v.25, p.88-97, 1996.
SATO, T.; WASHIZU, M.; KOBAYASHI, K. et al. Arrythmogenicity of dopamine and its effect on hemodynamics in dogs under halothane anesthesia. Nippon Juigaku Zasshi, v.51, p.396402, 1989.

STERNFELD, M. Acupunture \& Supraventricular tachicardia. Am. J. Acupunture., v.17, p.119-124, 1989.

TAYLOR, P.M. Stress responses in ponies during halothane or isoflurane anesthesia after induction with thiopentone or xylazine/ketamine. J. Vet. Anaesth., v.18, p.8-14, 1991.

TEIXEIRA NETO, F.J.; MASSONE, F.; LUNA, S.P. et al. The effects of atropine and methotrimeprazine on the epinephrine-induced arrhythmias in halotane-anestezed dogs. Can. J. Vet. Res., v.65, p.116-124, 2001.

UEDA, M.; MATSUMURA, S.; MATSUDA, S. et al. Comparative study between dobutamine and other catecholamines in their effects on the cardiac contraction and rythm. Nippon Yakurigaku Zasshi., v.73, p.501-516, 1977

YASHUDA, Y.; CAMARGO, L.A.A.; SAAD, W.A. Antiarrítmicos. In: ZANINI, A.C.; OGA, S. Farmacologia aplicada. 5.ed. São Paulo: Atheneu, 1994. p.306-315. 